\title{
The perception of vertical and horizontal distances in outdoor settings
}

\author{
ATSUKI HIGASHIYAMA and EIZO UEYAMA \\ University of Osaka Prefecture, Osaka, Japan
}

\begin{abstract}
In three experiments, perceived vertical and horizontal distances in outdoor settings were investigated. Horizontal distances were adjusted by 70 subjects to make them appear equal to vertical distances ranging from 2 to $47 \mathrm{~m}$. The results showed that (1) the matched horizontal distance is represented as a linear function of vertical distance; (2) the slope of the linear function is generally larger than unity, suggesting that when vertical distance is physically equal to horizontal distance, vertical distance appears larger than horizontal distance; (3) physiological muscular variables such as eye, head, and body position are not crucial in judging vertical and horizontal distances; (4) vertical distance of a building appears larger when viewed from afar than when viewed from nearby.
\end{abstract}

The purpose of this study was to determine whether, in outdoor settings, horizontal distance or vertical distance would appear to be longer. We refer to horizontal distance as the distance along the ground surface and vertical distance as the distance along the direction of gravity. An example of horizontal distance is the distance from an observer to a building, and an example of vertical distance is the height of the building.

Figure 1 shows four different viewing positions from which comparisons of vertical and horizontal distances can be made. Figure 1a illustrates the look-up condition, with the subject standing near the base of the building; Figure 1b illustrates the look-at condition, with the subject standing on the ground away from the building; Figure 1c illustrates the look-down condition, with the subject on the roof or a floor of the building looking down at the ground; Figure 1d illustrates the lie-down condition, with the subject lying on his/her back near the base of the building.

A number of studies have been performed under the look-up and lie-down conditions. Morinaga (1935) showed that for a vertical standard distance of $1 \mathrm{~m}$ in the look-up condition, the matched horizontal distance increased by $4 \%$ to $14 \%$ and did not change substantially with the position of eye and head. Makishita (1947, Experiment 1) determined equidistant curves as a function of elevation angle in the look-up condition, and showed that the matched vertical distance was smaller than the matched horizontal distance. Osaka (1947) demonstrated that for vertical standard distances of 4 to $12 \mathrm{~m}$, the matched horizontal distance increased by $12 \%$ to $21 \%$ in the lookup condition, but that this trend disappeared when the sub-

This paper was supported by a Grant-in-Aid for Scientific Researches (Shorei A: No. 62710072) provided by the Japanese Ministry of Education, Science, and Culture. Requests for reprints should be sent to Atsuki Higashiyama, Psychology Laboratory, University of Osaka Prefecture, Mozu-umemachi, Sakai, Osaka 591, Japan. ject lay down. Ohno $(1951,1972)$ used the horizontal standard and vertical comparison for the look-up condition and the vertical standard and horizontal comparison for the lie-down condition. The results showed that, for either condition, the matched distance was smaller than the standard. This implies that when vertical distance is physically equal to horizontal distance, vertical distance appears longer than horizontal distance for the look-up condition, but that the reverse is true for the lie-down condition. Galanter and Galanter (1973), using upright subjects, constructed psychophysical scales for perceived distance as a power function of physical distance, with the parameter being elevation angle. The results showed that the exponent of the power function decreased with increasing elevation angle, but was not influenced by such variables as background texture and the subject's motion. Ohno (1972), using upright subjects, obtained judgments of vertical and horizontal distances that were power functions of physical distance. The resulting exponents of the power function for vertical and horizontal distances did not differ, but the intercept was larger for vertical distance than for horizontal distance. Baird and Wagner (1982), with upright subjects estimating their apparent distances to the night sky, found that the sky at the zenith is generally estimated to be farther away than the sky at the horizon.

The results of these studies are so diverse as to defy concise summary. Furthermore, there is very little literature that is related to the look-at and look-down conditions. We could therefore make no predictions about the results of our own series of experiments.

In Experiment 1, we compared the look-up, look-at, and look-down conditions. In Experiment 2, we compared the lie-down condition with the look-up condition. For both experiments, a building and a courtyard were used for vertical distance and horizontal distance, respectively. To determine the generality of the results obtained from Experiments 1 and 2, Experiment 3 was performed in the 

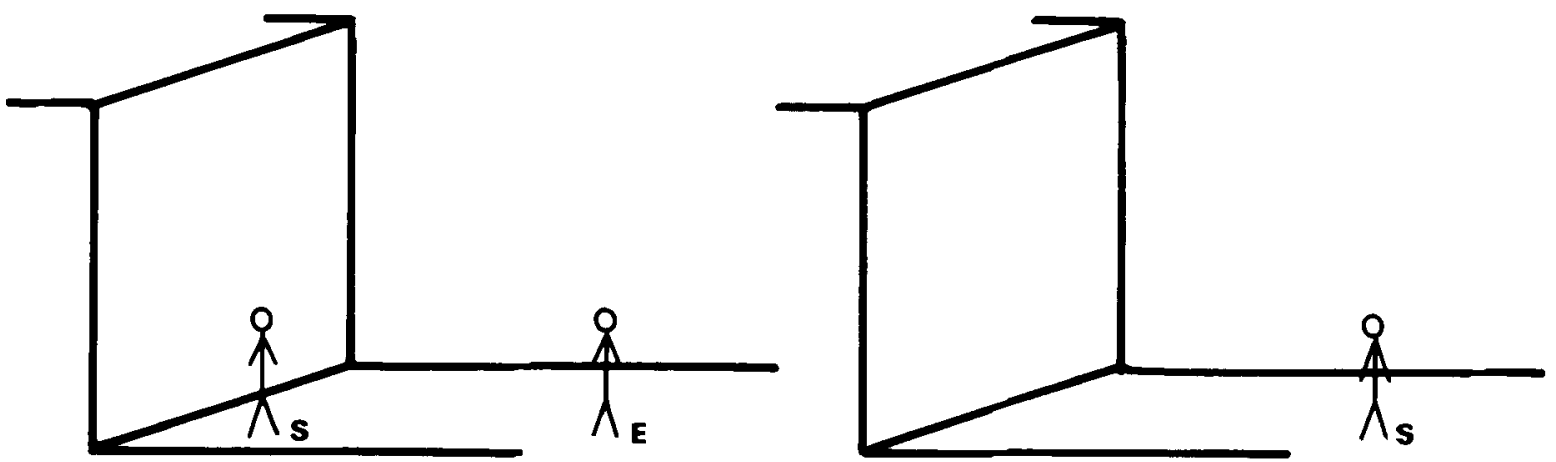

a. Look-up

b. Look-at
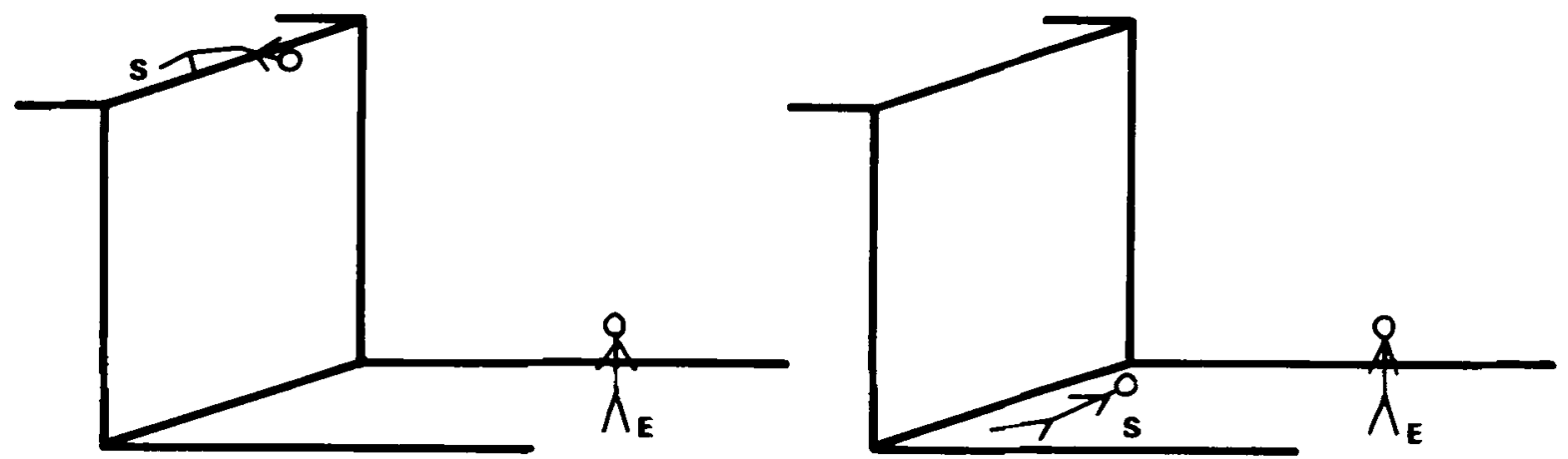

c. Look-down

d. Lie-down

Figure 1. Four viewing conditions: (a) the look-up condition; (b) the look-at condition; (c) the look-down condition; (d) the lie-down condition. The subject and experimenter are represented by $\mathbf{S}$ and $\mathbf{E}$, respectively.

look-at condition with 10 different targets (e.g., buildings, trees, and towers). In all experiments, horizontal distance was adjusted so that it appeared equal to vertical distance. In the look-at condition, the subject on the ground moved back and forth until the perceived horizontal distance from the subject to the building matched the perceived vertical distance of the building. In the lookup, look-down, and lie-down conditions, the experimenter on the ground moved back and forth in accordance with instructions from the subject.

\section{EXPERIMENT 1}

\section{Method}

Subjects. The subjects were 30 university students.

Experimental setting. The experiment was performed at a building of the College of Integrated Arts and Sciences at the university. This building bordered on the shorter side of a rectangular, approximately $45 \times 6.5 \mathrm{~m}$ courtyard. The outer wall of the building was covered with gray tiles $(5 \times 5 \mathrm{~cm})$, and five windows (67 cm wide $\times 190 \mathrm{~cm}$ high) were arranged vertically on the wall. The surface of courtyard was covered with white tiles $(30 \times 30 \mathrm{~cm})$. The top of the building was about $25 \mathrm{~m}$ above the ground.

The targets were five marks on the wall of the building: the four lower sides of the window frames and the roof of the building. Ver- tical distances from the ground to these marks were 3.0, 6.8, 10.6, 14.4 , and $18.7 \mathrm{~m}$, respectively. These marks were clearly visible to the subject on the ground.

Procedure. Each subject was assigned randomly to one of the look-up, look-at, and look-down groups, with the restriction that each group included 10 subjects.

In the look-up condition, the subject stood at a distance of $.5 \mathrm{~m}$ from the base of the building and adjusted horizontal distance so that the perceived horizontal distance was equal to each of the five perceived vertical distances. Horizontal distance was adjusted by having the experimenter approach or recede from the building. Because there was another gray building behind the experimenter, the subject could see it as the background of the experimenter. When vertical and horizontal distances appeared equal, the subject gave a stop sign to the experimenter. The distance between the building and the experimenter was then measured (with a tape measure). The subject made two distance adjustments, by approaching and backing away, for each mark on the vertical surface.

In the look-at condition, the same procedure as the look-up condition was repeated except that the subject adjusted horizontal distance by changing his/her position in the courtyard. In this condition, it was not necessary to use the experimenter as an adjustable point of reference for horizontal distance. The experimenter moved with the subject to measure the subject's distance from the building.

In the look-down condition, the same procedure as the look-up condition was repeated, except that the subject stood on the roof of the building or on the floor closest to each mark on the side wall 
of the building. The subject made comparative judgments of verti$\mathrm{cal}$ and horizontal distances and gave a stop sign to the experimenter on the ground. In this condition, the subject usually leaned over the window frame or roof railings to ensure a wide visual field of the courtyard. In each condition, the subject was allowed to move his/her eyes, head, and body.

\section{Results}

Figure 2 shows adjustments of horizontal distance against vertical distance. Each data point is a mean of $20(2 \times 10)$ adjustments. The parameter is the viewing condition.

A $3 \times 5 \times 2$ mixed-model analysis of variance (ANOVA), in which condition was a between-subjects factor and distance and trial were within-subjects factors, was performed on the adjustments of horizontal distance. There were significant main effects of condition $[F(2,27)$ $=14.37, p<.001]$ and of distance $[F(4,108)=488.55$, $p<.001]$. The interaction of condition with distance was also significant $[F(8,108)=6.91, p<.001]$. The simple main effects tests of the two-way interaction indicated that the main effects of condition were significant for vertical distances of $6.8 \mathrm{~m}[F(2,135)=3.17, p<.05]$,

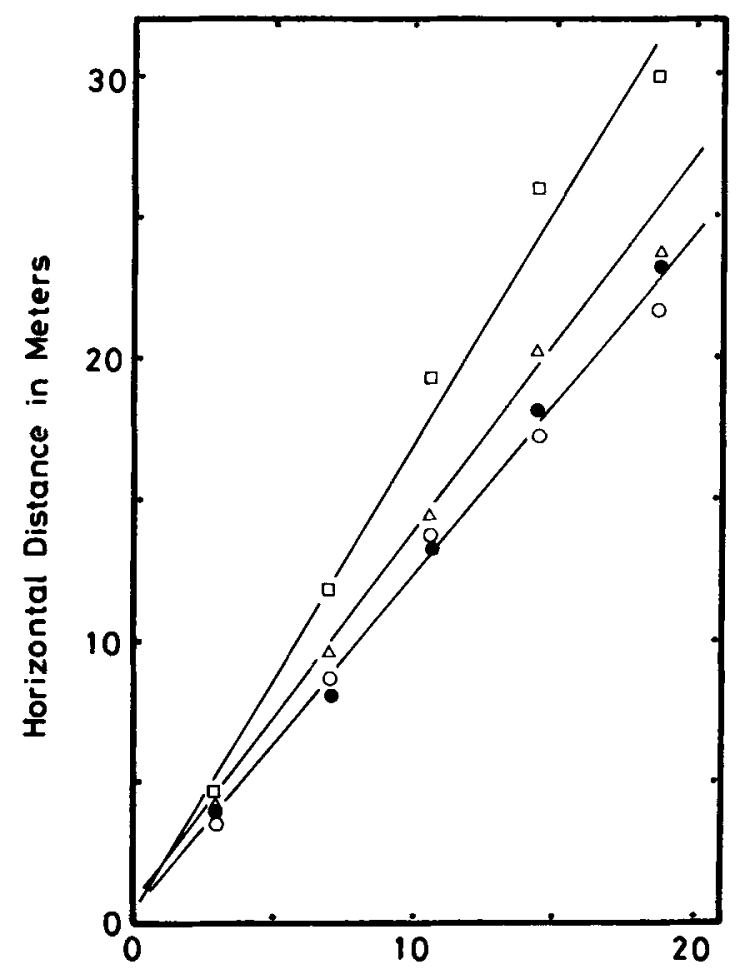

\section{Vertical Distance in Meters}

Figure 2. Matched horizontal distance as a function of vertical distance. The parameter is the viewing condition: Open circles are the look-up condition; squares are the look-at condition; triangles are the look-down condition; filled circles are the lie-down condition. For each of the look-up, look-at, and look-down conditions, $N=20$; for the lie-down condition, $N=40$.
Table 1

Parameter of Linear Functions Fitted to the Relations Between Vertical and Horizontal Distances

\begin{tabular}{|c|c|c|c|c|c|c|}
\hline \multirow{3}{*}{$\begin{array}{c}\text { Linear } \\
\text { Functions }\end{array}$} & \multicolumn{6}{|c|}{ Condition } \\
\hline & \multicolumn{2}{|c|}{ Look-up } & \multicolumn{2}{|c|}{ Look-at } & \multicolumn{2}{|c|}{ Look-down } \\
\hline & $M$ & $S D$ & $M$ & $S D$ & $M$ & $S D$ \\
\hline Slope & 1.16 & .27 & 1.67 & .29 & 1.26 & .14 \\
\hline Intercept & .61 & 1.23 & .57 & .98 & .98 & 1.38 \\
\hline$r^{2}$ & .939 & .046 & .955 & .032 & .938 & .061 \\
\hline
\end{tabular}

Note $-N=10$.

$10.6 \mathrm{~m}(F=10.63, p<.001), 14.4 \mathrm{~m}(F=21.82$, $p<.001)$, and $18.7 \mathrm{~m}(F=21.93, p<.001)$, but not significant for the vertical distance of $3.0 \mathrm{~m}(F<1)$.

The second analysis of data was based on the individual least squares lines fitted to the relation between vertical and horizontal distances for each viewing condition. The means and $S D$ s of slope, intercept, and coefficient of determination of the best-fitting linear functions are shown in Table 1. A one-way repeated measures ANOVA was performed on the slope data. The results showed that the main effect of condition was significant $[F(2,27)=10.98$, $p<.001]$. Tukey's $H S D$ tests indicated that the look-at condition produced a significantly steeper slope than the look-up $(p<.01)$ and look-down conditions $(p<.01)$, but that the difference between the look-up and look-down conditions was not significant. A series of $t$ tests indicated that the mean slopes were significantly larger than unity for the look-at condition $[t(9)=6.88, p<.001]$ and the look-down condition $[t(9)=5.54, p<.001]$.

Similarly, a one-way repeated measures ANOVA performed on the intercept data revealed that there was no significant main effect of condition. A series of $t$ tests showed that the mean intercept for any condition was not significantly different from zero.

\section{Discussion}

The matched horizontal distance $\left(D_{H}\right)$ is represented as a linear function of vertical distance $\left(D_{\mathrm{v}}\right)$; that is,

$$
D_{\mathbf{H}}=a D_{\mathbf{V}}+b \text {, }
$$

where $a$ and $b$ are constants. As shown in Table 1, this linear function accounted for about $94 \%$ of the variance in the average. This suggests that the linear function is fairly satisfactory for describing the relation between vertical and horizontal distances.

The linear function involves two independent parameters that should be distinguished conceptually and analytically. The slope $a$ denotes the degree of a precise match between vertical and horizontal distances. When $a$ is unity and $b$ is zero, a perfect match is obtained between the two distances. However, when $a$ is not unity and $b$ is zero, the adjustments deviate from the perfect match, in proportion to the vertical standard distance.

The intercept $b$, on the other hand, denotes a constant error, in the sense that the discrepancy between vertical 
and horizontal distances is consistently produced independently of vertical standard distance. This is illustrated by the fact that horizontal distance for a nonzero $b$ is equal to $b$ for vertical distance of zero.

The main results of Experiment 1 show that the value of slope is variable, depending on viewing condition, and the value of intercept is zero. This implies that the effects of viewing condition may be small for small vertical distance of less than $5 \mathrm{~m}$, but increase remarkably in size for longer vertical distance.

\section{EXPERIMENT 2}

With the results of Experiment 1, we found that the matched horizontal distances obtained in the look-up condition were not different from those in the look-down condition. This suggests that physiological muscular variables, such as head and eye position, do not greatly contribute to the judgments of vertical and horizontal distances. The purpose of Experiment 2 was to test this suggestion more directly. The subject lay down on the ground and matched the vertical distance to the horizontal distance. If the tension of muscle were the crucial variable in perceiving distance, the results of the lie-down condition would be different from those of the look-up condition of Experiment 1.

\section{Method}

Subjects. The subjects were 20 university students.

Procedure. Experiment 2 was performed in the same building and courtyard used in Experiment 1. The lie-down condition illustrated in Figure 1d was employed. The subject lay on his/her back on a thin mat, $.5 \mathrm{~m}$ from and parallel with the wall of the building. To view the marks on the wall, the subject kept his/her head straight; to see the experimenter in the courtyard, the subject was required to turn his/her head.

\section{Results and Discussion}

The results are shown in Figure 2 to facilitate comparison with the results of Experiment 1. The lie-down condition is marked with filled circles; each circle is a mean of $40(2 \times 20)$ adjustments. A best-fitting line according to a least squares criterion was obtained for each subject. The means of slope, intercept, and coefficient of determination were $1.25(S D=.25),-.06(S D=.95)$, and $.988(S D=.009)$, respectively. The mean slope was steeper than unity $[t(19)=4.35, p<.001]$, and the mean intercept was not significantly different from zero. Comparison of parametric values between the lie-down and look-up conditions showed that neither the difference of the mean slopes nor the difference of the mean intercepts was significant.

\section{EXPERIMENT 3}

The results of Experiments 1 and 2 showed that when vertical distance was matched by horizontal distance, horizontal distance was equal to (viz., the look-up condition) or larger than (viz., the look-at, look-down, and lie- down conditions) vertical distance. In the first two experiments, we did not find that the matched horizontal distance was smaller than the vertical standard distance. The purpose of Experiment 3 was to test the generality of these results, because Experiments 1 and 2 were both performed in the same, particularly organized setting of courtyard and building. In Experiment 3, the subject was asked to match the horizontal and vertical distances of 10 objects that were very different in height, color, shape, and background. The subject viewed the objects in the look-at condition.

\section{Method}

Subjects. The subjects were 20 university students.

Targets and Procedure. Ten objects on the campus were chosen as stimulus targets. The heights of those targets were directly measured by a tape or were estimated by a transit (Topcon MD-500A) with an accuracy of $1 \%$. To estimate the heights of objects that could not be measured directly, the experimenter faced each target and, using the transit, measured the distances from himself to the top $\left(D_{\mathrm{t}}\right)$ and bottom $\left(D_{\mathrm{b}}\right)$ of the target. The height $\left(D_{\mathrm{b}}\right)$ of the target was then computed on the basis of the Pythagorean theorem $\left[D_{\mathrm{h}}\right.$ $\left.=\left(D_{t}^{2}-D_{b}^{2}\right)^{1 / 2}\right]$. The targets (and their heights) were: three trees $(8.9,15.5$, and $18.5 \mathrm{~m}$, respectively), three buildings $(7.1,17.0$, and $27.3 \mathrm{~m}$, respectively), a power transmission tower $(47.5 \mathrm{~m})$, an electric light post $(13.0 \mathrm{~m})$, a telephone booth $(2.2 \mathrm{~m})$, and a monumental stone sculpture $(4.7 \mathrm{~m})$. These targets were dispersed throughout the campus and varied in color, shape, and background.

The main procedure was the same as that of the look-at condition of Experiment 1. By moving back and forth, the subject adjusted the horizontal distance until it appeared equal to the vertical distance (height) of each target. Vertical-distance adjustments were made for each target as in Experiment 1. The judgmental order of targets was randomized for each subject. Both the subject and the experimenter moved from target to target by bicycle.

\section{Results and Discussion}

For each subject, a least squares line was fitted to the matched horizontal distances as a function of vertical distance. The means of slope, intercept, and coefficient of determination were $1.62(S D=.30),-1.67(S D=2.82)$, and $.974(S D=.014)$, respectively. Figure 3 shows the mean linear function. Each data point is the mean of $40(2 \times 20)$ adjustments.

The mean slope was significantly steeper than unity $[t(19)=8.86, p<.001]$, and the mean intercept was significantly smaller than zero $[t(19)=2.59, p<.05]$. Comparison of parametric values between Experiment 3 and the look-at condition of Experiment 1 showed that the difference of the mean slopes was not significant, but the intercept was significantly smaller for Experiment 3 than for the look-at condition of Experiment $1[t(28)=$ $2.35, p<.05]$.

\section{GENERAL DISCUSSION}

The major findings of this study are that the matched horizontal distance was represented as a linear function of vertical standard distance and the slope of the linear function was generally larger than unity. This suggests that when vertical distance is physically equal to horizontal 


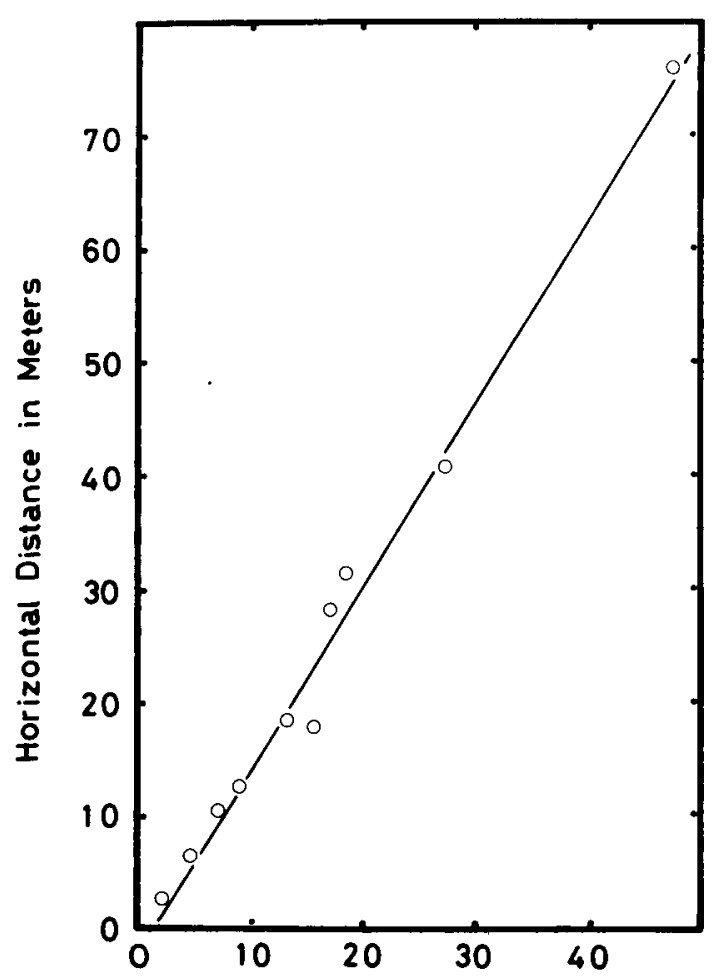

Vertical Distance in Meters

Figure 3. Matched horizontal distance as a function of vertical distance for 10 different targets under the bok-at condition. $N=\mathbf{4 0}$.

distance, the vertical distance appears larger than the horizontal distance. These results support those of Morinaga (1935) and Makishita (1947) but contradict those of Galanter and Galanter (1973).

It is of interest that the mean slopes of the linear function were not significantly different between the look-up and look-down conditions or between the look-up and liedown conditions. This means that the positions of eye, head, and body are not crucial in the perception of distance. These results confirm those of Morinaga (1935) and Galanter and Galanter (1973) but contradict those of Ohno (1951, 1972) and Osaka (1947).

\section{Scales for Perceived Distance}

A number of studies (Baird \& Wagner, 1982; Cook, 1978; Da Silva, 1983, 1985; Galanter \& Galanter, 1973; Kuroda, 1971; Ohno, 1972; M. Teghtsoonian \& R. Teghtsoonian, 1969; R. Teghtsoonian \& M. Teghtsoonian, 1970; Ueno, 1962) have described perceived horizontal distance $\left(D_{\mathbf{H}}^{\prime}\right)$ as a power function of physical horizontal distance:

$$
D_{\mathbf{H}}^{\prime}=k D_{\mathbf{H}}^{n},
$$

where $k$ and $n$ are constants. In this study, on the other hand, vertical distance was matched by horizontal distance, so that

$$
D_{\mathbf{V}}^{\prime}=D_{\mathbf{H}}^{\prime},
$$

where $D_{v}^{\prime}$ is perceived vertical distance.

From Equations 2 and 3, we obtain

$$
D_{\mathbf{v}}^{\prime}=k D_{\mathbf{H}}^{n} \text {. }
$$

From Equations 1 and 4, furthermore, we obtain

$$
D_{v}^{\prime}=k\left(a D_{v}+b\right)^{n} \text {. }
$$

Since the parameter $b$ is equal to zero (Experiments 1 and 2 ) or is very close to zero (Experiment 3), Equation 5 is approximated by

$$
D_{\mathbf{v}}^{\prime}=K D_{\mathbf{v}}^{n}
$$

where $K=k a^{n}$.

Thus, it is suggested that (1) perceived vertical and horizontal distances are described as a power function of physical vertical and horizontal distances, respectively, (2) the exponent of the power function is the same for both distance directions, and (3) the scale factor for vertical distance is equal to or larger than that for horizontal distance, because when $a \geq 1, k\left(a^{n}-1\right) \geq 0$. These suggestions are in agreement with the results of Ohno (1972) but not with those of Galanter and Galanter (1973).

\section{Texture Gradient of Vertical and Horizontal Surfaces}

The slopes for the look-at condition were steeper than those for the other three conditions. What distinguishes the look-at condition from the other conditions lies in the visual angle that an object on a vertical surface forms at the subject's eye position. The small insert in the top right of Figure 4 illustrates the geometric relationships of an object on a vertical surface, the subject's position, and visual angle. To demonstrate the relationship systematically, Figure 4 shows the visual angle $(\theta)$ of a 1 -m-long stick as a function of vertical distance, with the parameter being the horizontal distance from the vertical surface to the subject. A set of curves in Figure $\mathbf{4}$ was obtained from the equation

$$
\begin{aligned}
\theta & =\theta_{\mathrm{t}}-\theta_{\mathrm{b}} \\
& =57.3\left(\arctan \frac{D_{\mathbf{V}}}{D_{\mathbf{H}}}-\arctan \frac{D_{\mathbf{V}}-1}{D_{\mathbf{H}}}\right),
\end{aligned}
$$

where $\theta_{\mathrm{t}}$ and $\theta_{\mathrm{b}}$ are visual angles in degrees that are measured from the horizontal eye level to the top and bottom of the stick, respectively.

Figure 4 indicates that (1) the shorter the horizontal distance, the more rapidly the visual angle of the stick decreases as a function of vertical distance, and (2) for a vertical distance of about $5 \mathrm{~m}$ or more from the horizontal eye level, the visual angle of the stick is generally larger for the look-at condition $\left(D_{\mathrm{H}}=2.5\right.$ to $\left.40 \mathrm{~m}\right)$ than for the look-up and lie-down conditions $\left(D_{\mathrm{H}}=.5 \mathrm{~m}\right)$, but that for a vertical distance of less than $2 \mathrm{~m}$, the reverse is true. In other words, for the look-up and lie-down conditions, texture on the wall of the building is compressed rapidly 


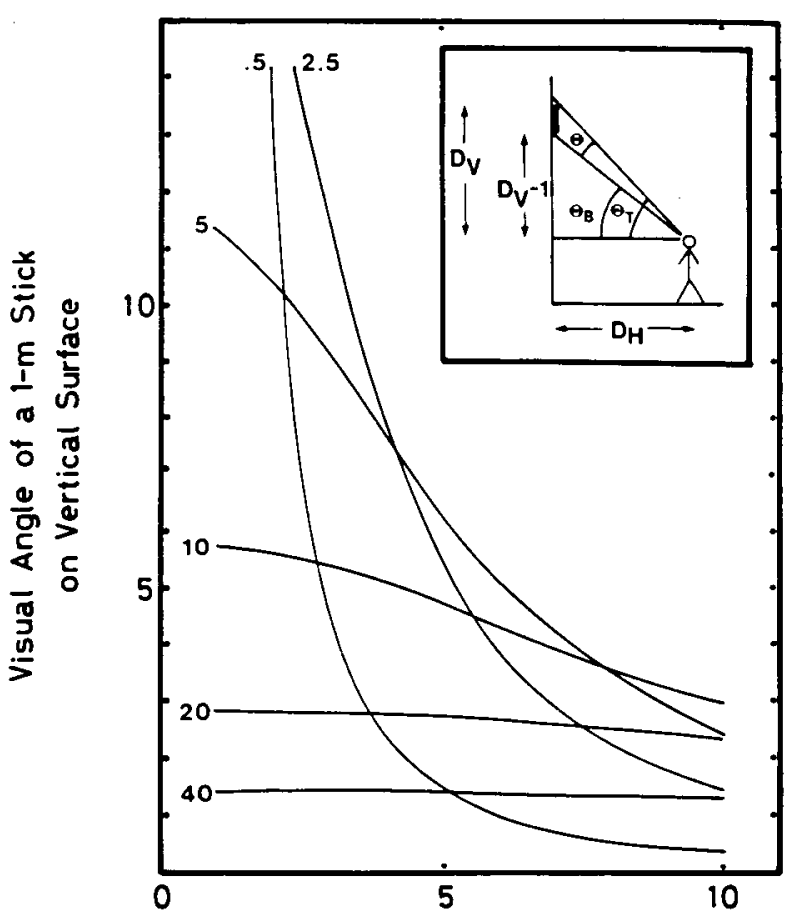

Vertical Distance in Meters

Figure 4. Visual angle in degrees of a 1-m stick on a vertical surface as a function of vertical distance. The parameter is the horizontal distance in meters from the vertical surface to the subject.

as a function of vertical distance and may disappear near the top of the building, whereas for the look-at condition, the texture gradient is so slow that vertical distance is discriminated even at the top of the building. Therefore, vertical distance is probably judged to be longer in the lookat condition than in the other conditions.

This view also applies to the texture of the ground; that is, rapid compression of the texture of the ground is expected when subjects are on the ground. For subjects above the ground, however, the texture gradient of the ground is slower. Therefore, look-down subjects' judgments of horizontal distance may be longer than those of the subjects in the other conditions.

If the perceived-distance matches are made on the basis of these differences of texture gradient between vertical and horizontal directions, it is predicted that the longest adjustments of horizontal distance will be obtained for the look-at condition, whereas the shortest adjustments will be obtained for the look-down condition. The results of Figure 2 partially support this prediction, because the look-at condition produced the longest adjustments among the four conditions, but the adjustments of the look-down condition did not produce the smallest adjustments.

Because the difference of texture gradient between vertical and horizontal directions may be found at relatively far distances, the discrepant matches between vertical and horizontal distances would not be produced at short distances. In fact, the results of Experiment 1 showed that the effects of viewing position were not found at a vertical distance of less than $5 \mathrm{~m}$.

\section{Stability and Veridicality}

It should be noted that perceived distance is very stable outdoors. The mean slope (1.67) obtained in the lookat condition of Experiment 1 was very close to the mean slope (1.62) obtained in Experiment 3, despite the differences of target, stimulus range, and background. The idea of stability is also supported by the finding that perceived distance was not influenced by the positions of eye, head, and body. However, it is clear that perceived distance is not precise even in the full-cue situation, because the matched horizontal distance is generally greater than the vertical standard distance, as shown in this study. It is therefore suggested that the richness of distance cues contributes to the stability of perceived distance but does not necessarily lead to the veridicality of perceived distance.

\section{REFERENCES}

Baird, J. C., \& Wagner, M. (1982). The moon illusion: How high is the sky? Journal of Experimental Psychology: General, 111, 296-303.

Cook, M. (1978). The judgment of distance on a plane surface. Perception \& Psychophysics, 23, 85-90.

Da Silva, J. A. (1983). Ratio estimation of distance in a large open field. Scandinavian Journal of Psychology, 24, 343-345.

DA SilvA, J. A. (1985). Scales for perceived egocentric distance in a large open field: Comparison of three psychophysical methods. American Journal of Psychology, 98, 119-144.

Galanter, E., \& Galanter, P. (1973). Range estimates of distant visual stimuli. Perception \& Psychophysics, 14, 301-306.

KuroDA, T. (1971). Distance constancy: Functional relationships between apparent distance and physical distance. Psychologische Forschung, 34, 199-219.

MAkISHITA, S. (1947). Gensho-teki tokyori kukan ni kansuru sho-jikken [Experiments on apparent equidistances]. Shinri, 1, 62-64.

MoRINAGA, S. (1935). Shi-hoko to tsuki no sakushi [Visual direction and the moon illusion]. Japanese Journal of Psychology, 10, 1-25.

OHNo, S. (1951). Chikaku-teki kukan no hi-toshitsusei ni kansuru jikkenteki kenkyu [An experimental study on inhomogeneity of perceptual space]. Saga Daigaku Kyoiku Gakubu Ronbunshu, 1, 75-95.

OHNO, S. (1972). Chikaku kukan no ihosei [Anisotropy of perceptual space]. In Y. Akishige (Ed.), Chikaku-teki sekai no kojosei. Ninshiki shinrigaku [Constancies of perceptual world: Epistemological psychology] (Vol. 4, pp. 387-575). Tokyo: Ibunsha.

OsAKA, R. (1947). Shi-kukan no ihosei-tsuki no sakushi ni tsuite [Anisotropy of visual space: The moon illusion]. Shinri, 1, 65-98.

Teghtsoonian, M., \& Teghtsoonian, R. (1969). Scaling apparent distance in natural indoor settings. Psychonomic Science, 16, 281-283.

Teghtsoonian, R., Teghtsioonian, M. (1970). Scaling apparent distance in a natural outdoor setting. Psychonomic Science, 21, 215-216.

UENo, T. (1962). The size-distance invariance hypotheses and psychophysical law. Japanese Psychological Research, 4, 99-112.

(Manuscript received August 31, 1987; revision accepted for publication January 11, 1988.) 